\title{
Guideline update for the performance of fusion procedures for degenerative disease of the lumbar spine. Part 17: Bone growth stimulators as an adjunct for lumbar fusion
}

\author{
Michael G. Kaiser, M.D., ${ }^{1}$ Jason C. Eck, D.O., M.S., ${ }^{2}$ Michael W. Groff, M.D., ${ }^{3}$ \\ Zoher Ghogawala, M.D., ${ }^{4}$ William C. Watters III, M.D., ${ }^{5}$ Andrew T. DAiley, M.D., 6 \\ Daniel K. Resnick, M.D., 7 Tanvir F. Choudhri, M.D., 8 Alok Sharan, M.D., 9 \\ Jeffrey C. Wang, M.D., ${ }^{10}$ Sanjay S. Dhall, M.D., ${ }^{11}$ and Praveen V. Mummaneni, M.D. ${ }^{11}$ \\ ${ }^{1}$ Department of Neurosurgery, Columbia University, New York, New York; ${ }^{2}$ Center for Sports Medicine and \\ Orthopaedics, Chattanooga, Tennessee; ${ }^{3}$ Department of Neurosurgery, Brigham and Women's Hospital, \\ Boston, Massachusetts; ${ }^{4}$ Alan and Jacqueline Stuart Spine Research Center, Department of Neurosurgery, \\ Lahey Clinic, Burlington, and Tufts University School of Medicine, Boston, Massachusetts; ${ }^{5}$ Bone and Joint \\ Clinic of Houston, Houston, Texas; ${ }^{6}$ Department of Neurosurgery, University of Utah, Salt Lake City, Utah; \\ ${ }^{7}$ Department of Neurosurgery, University of Wisconsin, Madison, Wisconsin; ${ }^{8}$ Department of Neurosurgery, \\ Icahn School of Medicine at Mount Sinai, New York, New York; ${ }^{9}$ Department of Orthopaedic Surgery, \\ Montefiore Medical Center, Albert Einstein College of Medicine, Bronx, New York; ${ }^{10}$ Department of \\ Orthopaedic Surgery, Keck School of Medicine, University of Southern California, Los Angeles, California; \\ and ${ }^{11}$ Department of Neurological Surgery, University of California, San Francisco, California
}

The relationship between the formation of a solid arthrodesis and electrical and electromagnetic energy is well established; most of the information on the topic, however, pertains to the healing of long bone fractures. The use of both invasive and noninvasive means to supply this energy and supplement spinal fusions has been investigated. Three forms of electrical stimulation are routinely used: direct current stimulation (DCS), pulsed electromagnetic field stimulation (PEMFS), and capacitive coupled electrical stimulation (CCES). Only DCS requires the placement of electrodes within the fusion substrate and is inserted at the time of surgery. Since publication of the original guidelines, few studies have investigated the use of bone growth stimulators. Based on the current review, no conflict with the previous recommendations was generated. The use of DCS is recommended as an option for patients younger than 60 years of age, since a positive effect on fusion has been observed. The same, however, cannot be stated for patients over 60 , because DCS did not appear to have an impact on fusion rates in this population. No study was reviewed that investigated the use of CCES or the routine use of PEMFS. A single low-level study demonstrated a positive impact of PEMFS on patients undergoing revision surgery for pseudarthrosis, but this single study is insufficient to recommend for or against the use of PEMFS in this patient population.

(http://thejns.org/doi/abs/10.3171/2014.4.SPINE14326)

\section{KEY WORDS • lumbar spine • lumbar fusion • bone growth stimulator • practice guidelines}

\section{Recommendations}

There is no evidence that conflicts with the previous recommendations regarding bone growth stimulation published in the original version of the "Guidelines for the performance of fusion procedures for degenerative disease of the lumbar spine."18

\footnotetext{
Abbreviations used in this paper: $\mathrm{BMP}=$ bone morphogenetic protein; CCES = capacitive coupled electrical stimulation; DCS = direct current stimulation; DEXA = dual energy x-ray absorptiometry; DPQ = Dallas Pain Questionnaire; LBPRS = Low Back Pain Rating Scale; PEMFS = pulsed electromagnetic field stimulation; SF-36 = 36-Item Short Form Health Survey; VAS = visual analog scale.
}

\section{Grade $C$}

The routine use of DCS in patients over the age of 60 years is not recommended, as the evidence demonstrates no impact on fusion rates (single Level II study).

For patients younger than 60 years of age, undergoing a lumbar fusion, the use of DCS is an option as studies have demonstrated a positive impact on fusion rate; however, there is insufficient evidence regarding its impact on clinical outcome (single Level III study/multiple Level IV studies).

\section{Grade I}

There is insufficient evidence to recommend for or against the use of PEMFS as a treatment alternative to re- 
vision surgery in patients presenting with pseudarthrosis following posterior lumbar fusion (single Level IV study).

\section{Rationale}

Since the publication of the original "Guidelines for the performance of fusion procedures for degenerative disease of the lumbar spine,"16 the evidence supporting the role of lumbar fusion as an effective treatment alternative for a variety of degenerative spinal conditions continues to expand. ${ }^{6,9,12,22}$ As the role of lumbar fusion becomes more established, increasing emphasis has been placed on maneuvers to enhance the potential for a solid arthrodesis. The positive impact of spinal instrumentation on fusion rates is well recognized..$^{5,11,17}$ There is also a growing body of evidence demonstrating a beneficial effect on fusion rates with osteoinductive agents. ${ }^{1,7,8}$ The data supporting the role of bone growth stimulators remain inconclusive and more controversial..$^{15,18}$

The interaction between electrical energy and the formation of an osseous union is a well-recognized concept, with the majority of clinical data focusing on long bone healing..$^{15}$ Dwyer published one of the first manuscripts describing the utilization of direct current stimulation (DCS) for spinal fusion. ${ }^{10}$ Since this report, 3 forms of electrical stimulation have gained acceptance for use in spinal fusion: DCS, pulsed electromagnetic field stimulation (PEMFS), and capacitive coupled electrical stimulation (CCES). DCS requires the insertion of cathodes, attached to an implanted battery, directly into the fusion substrate. PEMFS is a noninvasive means of delivering electromagnetic energy to the fusion by wearing an external coil driven by an electrical current. CCES relies on the generation of an electrical field through capacitive plates placed on the patient's skin. ${ }^{15}$ The purpose of this update was to review the current literature and examine the evidence supporting the clinical utility of various bone growth stimulators for lumbar fusion surgery, although no studies investigating the efficacy of CCES were identified.

\section{Search Criteria}

A computerized search of the National Library of Medicine MEDLINE database, utilizing the online search engine PubMed, was conducted for the period from 2003 through December 2011 utilizing the following search terms ((“"Lumbosacral Region”[MeSH] OR "Lumbar Vertebrae"[MeSH]) AND "Spinal Fusion" [MeSH]) OR "lumbar fusion"[All Fields] OR ("lumbar" [title] AND "fusion"[title])) AND ((bone growth stimulator[title] OR bone growth stimulators[title]) OR ("Electric Stimulation"'[MeSH] OR "Electric Stimulation Therapy"[MeSH] OR (("bone and bones"[MeSH] OR ("bone"[All Fields] AND "bones"[All Fields]) OR "bone and bones"[All Fields] OR "bone"[All Fields]) AND stimulator[All Fields]) OR ((“bone and bones"[MeSH] OR ("bone"[All Fields] AND "bones"[All Fields]) OR "bone and bones"[All Fields] OR "bone"[All Fields]) AND stimulators[All Fields]))). The search was limited to the English language and human subjects and yielded a total of 44 articles. The titles and abstracts of these publications were reviewed and those specifically investigating the clinical efficacy of bone growth stimulation were selected. A secondary review of the bibliographies of these articles was conducted to identify any additional relevant manuscripts. A total of 5 manuscripts were selected and serve as the scientific foundation for the updated review.

\section{Scientific Foundation}

Andersen et al. performed a randomized, controlled, multicenter trial to determine the impact of DCS on functional outcome of noninstrumented lumbar fusion for patients over 60 years of age. ${ }^{3}$ One hundred seven patients presenting with a variety of spinal degenerative disorders and undergoing single or multilevel posterolateral lumbar fusion (PLF) with local autograft and allograft were randomized into cohorts with a 40-mA $(\mathrm{n}=44)$ or $100-\mathrm{mA}(\mathrm{n}$ =11) DCS implanted stimulator or without $(n=43)$ DCS. For a variety of reasons, 9 randomized patients were excluded either prior to surgery or due to intraoperative complications. Patients completed a series of validated, objective outcome instruments (the 36-Item Short Form Health Survey [SF-36], the Dallas Pain Questionnaire [DPQ], and the Low Back Pain Rating Scale [LBPRS]), and statistical analysis was performed to compare treatment effect. Patients were followed up for 2 years; however, 27\% of patients did not complete the functional outcome questionnaires at this end point. At the 2-year point, the patients in the combined treatment group demonstrated significantly greater improvement in 3 of the 4 domains of the DPQ, although no significant difference in LBPRS or SF-36 scores was observed. Based on these results the authors concluded that surgery led to an improvement in functional outcome and that DCS may have a beneficial effect on lumbar fusion in older patients. This is a relatively well-designed randomized trial, but the study does suffer from several limitations. The validity of separating the results of the individual domains within the DPQ is unclear because the overall percentages are graphed to create a profile summary of the patient. ${ }^{14}$ Variability existed with respect to the presenting diagnosis and surgical intervention. It is not clear who performed the functional assessment and whether that individual was blinded to the treatment received. At the 2-year follow-up, only $73 \%$ of the participants completed the functional assessment questionnaires. Finally, the statistical analysis was limited by the authors' failure to determine the confidence intervals for the observed results. Due to these limitations the study was downgraded to Level II evidence supporting the role of DCS for this patient population undergoing noninstrumented lumbar fusion (Table 1).

Anderson and colleagues published 2 additional studies based on the same patient population with the intention of determining the effect of DCS on fusion rate, correlating the radiographic outcome to clinical outcome, and clarifying whether DCS had an impact on the quality of fusion..$^{2,4}$ Of the original 107 patients randomized, 95 were available for fusion assessment at 1 year and 84 were available at 2 years. Thin-slice CT images and plain radiographs were used to assess fusion status. In both the control and treatment cohorts the observed fusion rate was 
Part 17: Bone growth stimulators as an adjunct for lumbar fusion

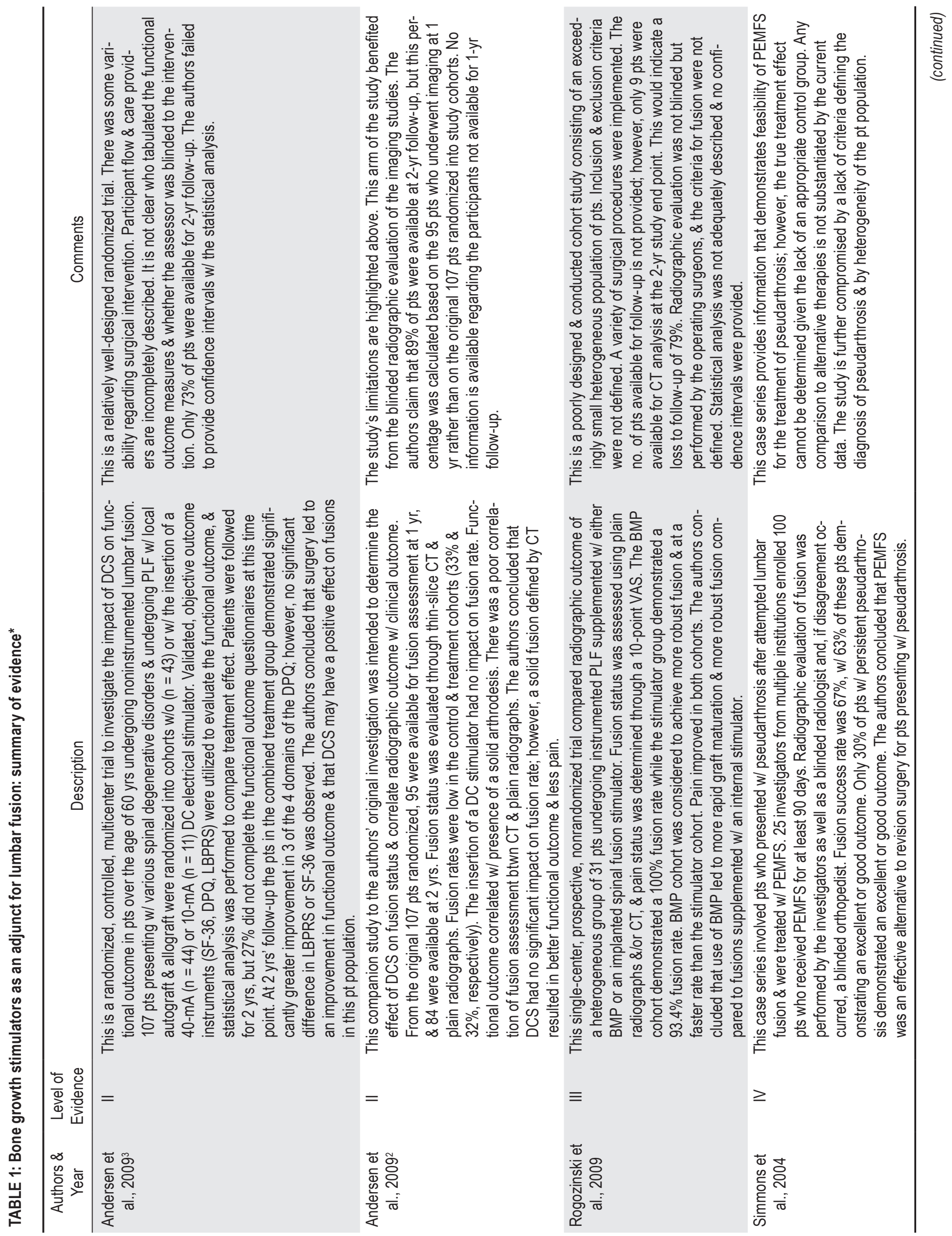


M. G. Kaiser et al.

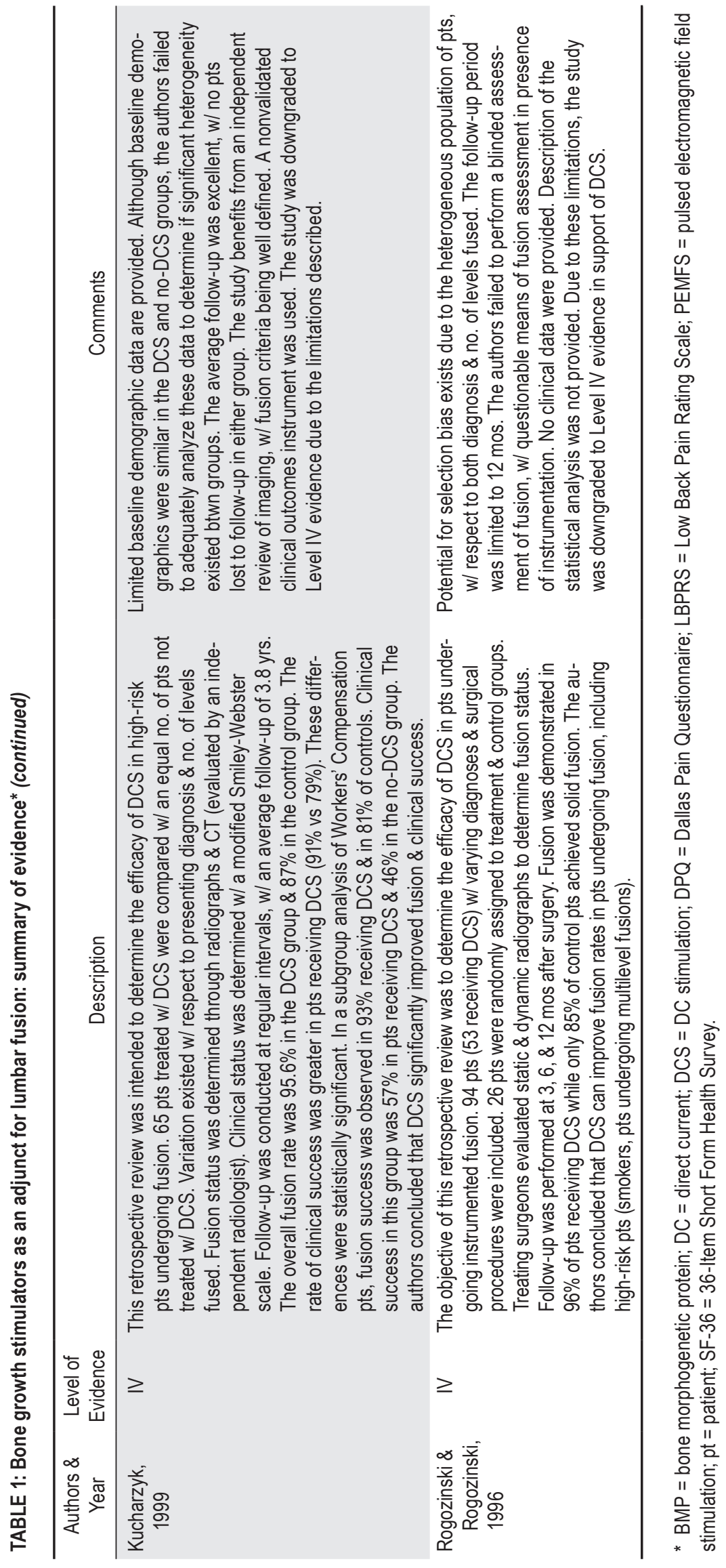


low-33\% and $32 \%$, respectively. The authors concluded that the utilization of DCS had no impact on fusion rate. There was a poor correlation between the observations made from CT images and plain radiographs, although a solid fusion, as defined by CT, resulted in better functional outcome and less pain ${ }^{2}$ (Table 1). The final study from this series demonstrated, through the use of dual energy x-ray absorptiometry (DEXA), that the application of DCS had no impact on the bone mineral density of the fusion mass. ${ }^{4}$ These investigations suffer from the same limitations as the first study in this series. The blinded radiographic assessment of the $\mathrm{CT}$ imaging strengthens the observations and conclusions regarding the impact of DCS on fusion rate. Although the authors claim that there was an $89 \%$ follow-up rate at 2 years, this was calculated from the 95 patients undergoing imaging at 1 year and not from the original 107 patients randomized at the onset of the study. Like the original study, this investigation was downgraded and provides Level II evidence against the utility of DCS to enhance the fusion rate for noninstrumented lumbar fusion. In a follow-up study, Andersen et al. investigated the impact of DCS on the quality of fusion formation by examining 80 of the original 107 patients with DEXA at 1 year after surgery. No significant difference in bone mineral density was observed between the 3 treatment groups.

Rogozinski et al. conducted a prospective, nonrandomized trial comparing radiographic outcome in 31 patients with the diagnosis of degenerative disc disease, who underwent 1- to 3-level instrumented PLF supplemented with either bone morphogenetic protein (BMP) or an implanted DC stimulator. ${ }^{20}$ Fusion status was assessed using plain radiographs and/or CT, and pain status was determined through a 10-point visual analog scale (VAS). The BMP cohort demonstrated a $100 \%$ fusion rate, while the stimulator group demonstrated a $93.4 \%$ fusion rate. The BMP cohort was considered to achieve more robust fusion at a faster rate than the stimulator cohort. Pain improved in both cohorts. The authors concluded that the use of BMP led to more rapid graft maturation and a more robust fusion compared with fusions supplemented with an internal stimulator. The actual treatment effect of DCS compared with traditional fusion techniques cannot be determined from this study because all patients received some form of fusion supplement, but the fusion rate observed in the DCS cohort is comparable to previously reported rates of fusion for similar patients without DCS. This investigation also suffers from major limitations with respect to study design, including a small, heterogeneous patient cohort, lack of inclusion and exclusion criteria, and heterogeneous surgical treatments. Only 9 patients were available for CT imaging at 2 years after surgery (79\% lost to follow-up), the assessment of the images was performed by the treating surgeon, and the criteria for fusion were not defined. This study was therefore downgraded to Level III evidence, although one may consider it simply a case series with respect to the DCS data (Table 1).

Two additional studies have also demonstrated a positive impact of DCS on fusion formation. Kucharzyk performed a retrospective review of 130 cases involving patients undergoing lumbar fusion with $(n=65)$ and with- out $(n=65)$ placement of DCS. ${ }^{13}$ Fusion status was determined through both $\mathrm{CT}$ images and plain radiographs. The average follow-up was 3.8 years. The fusion rate in the DCS cohort was $95.6 \%$, while the rate in the control group was $87 \%$. Clinical success, utilizing a nonvalidated outcome measure, was superior in the DCS group. Rogozinski and Rogozinski also performed a retrospective review of 94 cases, with 53 of the patients receiving a DCS, and observed a fusion rate of $96 \%$ in the DCS cohort and $85 \%$ in the control arm. ${ }^{19}$ Both of these studies suffer from a heterogeneous population of patients, limited baseline demographic data, and either failure to report clinical outcome or use of a nonvalidated instrument. Due to these limitations these studies are downgraded to Level IV evidence in support of the use of DCS with lumbar fusions.

Simmons et al. published a case series involving 100 patients with a mean age of 43.3 years who presented with pseudarthrosis after an attempt at single- or multilevel lumbar fusions and were treated with pulsed electromagnetic field stimulation (PEMFS). ${ }^{21}$ Pseudarthrosis was confirmed by the presence of motion on dynamic imaging and the lack of visible bone healing on CT, MRI, or radiographic images. Twenty-five investigators from multiple institutions enrolled the 100 patients, who received PEMFS for at least 90 days. The investigators as well as a blinded radiologist performed radiographic evaluation of fusion. If there was disagreement among reviewers, an independent evaluation was performed by a blinded orthopedist. A solid fusion was defined as $50 \%$ or more assimilation of the graft based on radiographic imaging; the specific imaging technique was not defined. Clinical outcome was rated as excellent, good, fair, or poor, based on patients' reported pain intensity, medication usage, and return to work. The fusion success rate was $67 \%$, and $63 \%$ of the patients with successful fusion demonstrated an excellent or good outcome. Only $30 \%$ of patients with persistent pseudarthrosis had an excellent or good outcome. The authors concluded that PEMFS was an effective alternative to revision surgery for patients presenting with pseudarthrosis. Although this study provides evidence that the utilization of PEMFS is a feasible intervention for the management of pseudarthrosis, the true treatment effect cannot be determined due to the study design and lack of an adequate control group. The authors also fail to define the criteria used to diagnose pseudarthrosis and included a heterogeneous population of patients. The study therefore provides at best Level IV evidence in support of PEMFS for treatment of pseudarthrosis (Table 1).

\section{Summary}

Based on the recommendations from the original guidelines, both DCS and CCES may be considered in patients at high risk for pseudarthrosis who are undergoing PLF, while PEMFS may be considered in a similar patient population undergoing an interbody fusion. Since the publication of the previous guidelines, there have been few clinical trials that provide further insight into the clinical utility of bone growth stimulation. The current data do not contribute to the previous recommendations. 
The few studies that have investigated the use of bone growth stimulators have methodological flaws that compromise the conclusions and prohibit the formulation of strong recommendations. Based on a single Level II study, there is a suggestion that the use of DCS in patients over 60 years of age may provide a clinical benefit; however, this benefit was only observed in a subset of measures from a single outcome instrument and therefore is considered a weak correlation. This potential beneficial effect is further weakened by the fact that DCS did not have a positive impact on the fusion rate or quality in the same patient population. The weak correlation to clinical outcome may therefore be an artifact of the flawed study design or simply due to chance. Since the intended purpose of DCS was not supported by the authors' observations, the routine use of DCS in patients over 60 years of age undergoing a noninstrumented fusion was not recommended.

The second recommendation supports the use of PEMFS in patients suffering from a pseudarthrosis, but no comment can be made regarding the routine use of PEMFS. Due to the noninvasive nature of PEMFS, its application appears to be relatively benign with few drawbacks; however, in today's medical climate one cannot ignore the costs associated with an intervention that has not been proven to provide definitive benefit. Unfortunately, the quality of the current literature does not help to address these concerns.

\section{Key Issues for Future Investigation}

The impact of bone growth stimulators on fusion rates is likely to be minimal, and this makes it difficult to conduct a clinical trial to determine the actual treatment effect and/or compare the efficacy of different types of stimulators. Given the noninvasive nature of PEMFS, a well-designed randomized controlled trial is feasible. Such a study would, however, require an exceedingly large number of patients to demonstrate the difference in treatment effect. Nevertheless, such information would prove valuable, not only from a clinical perspective, but also for effective cost analysis, which ultimately may be the more relevant issue in today's medical climate. Utilization of a prospective patient registry may also provide relevant information by identifying specific patient populations that would benefit from any advantage provided by fusion enhancers, such as bone growth stimulators.

\section{Acknowledgments}

We would like to acknowledge the AANS/CNS Joint Guidelines Committee (JGC) for their review, comments, and suggestions; Laura Mitchell, CNS Guidelines Project Manager, for her organizational assistance; and Linda O'Dwyer, medical librarian, for assistance with the literature searches. We would also like to acknowledge the following individual JGC members for their contributions throughout the review process: Timothy Ryken, M.D.; Kevin Cockroft, M.D.; Sepideh Amin-Hanjani, M.D.; Steven N. Kalkanis, M.D.; John O'Toole, M.D., M.S.; Steven Casha, M.D., Ph.D.; Aaron Filler, M.D., Ph.D., F.R.C.S.; Daniel Hoh, M.D.; Steven Hwang, M.D.; Todd McCall, M.D.; Jeffrey J. Olson, M.D.; Julie Pilitsis, M.D., Ph.D.; Joshua Rosenow, M.D.; and Christopher Winfree, M.D.

\section{Disclosure}

Administrative costs of this project were funded by the Congress of Neurological Surgeons and the Joint Section on Disorders of the Spine and Peripheral Nerves of the American Association of Neurological Surgeons and Congress of Neurological Surgeons. No author received payment or honorarium for time devoted to this project. Dr. Ghogawala receives grants from the Patient Centered Outcomes Research Institute (PCORI) and the National Institutes of Health (NIH). Dr. Groff is a consultant for DePuy Spine and EBI Spine. Dr. Mummaneni owns stock in Spinicity and receives honoraria from DePuy Spine and Globus and royalties from DePuy Spine, Quality Medical Publishers, and Thieme Publishing. Dr. Wang owns stock in Bone Biologics, AxioMed, Amedica, CoreSpine, Expanding Orthopedics, Pioneer, Syndicom, VG Innovations, PearlDiver, Flexuspine, Axis, FzioMed, Benvenue, Promethean, Nexgen, ElectroCore, and Surgitech and holds patents with and receives royalties from Biomet, Stryker, SeaSpine, Aesculap, Osprey, Amedica, Synthes, and Alphatec. The authors report no other potential conflicts of interest concerning the materials or methods used in this study or the findings specified in this paper.

Author contributions to the study and manuscript preparation include the following. Acquisition of data: all authors. Analysis and interpretation of data: all authors. Drafting the article: Kaiser. Critically revising the article: all authors. Reviewed submitted version of manuscript: all authors. Approved the final version of the manuscript on behalf of all authors: Kaiser. Study supervision: Kaiser.

\section{References}

1. Agarwal R, Williams K, Umscheid CA, Welch WC: Osteoinductive bone graft substitutes for lumbar fusion: a systematic review. Clinical article. J Neurosurg Spine 11:729-740, 2009

2. Andersen T, Christensen FB, Egund N, Ernst C, Fruensgaard S, Østergaard J, et al: The effect of electrical stimulation on lumbar spinal fusion in older patients: a randomized, controlled, multi-center trial. Part 2: fusion rates. Spine (Phila Pa 1976) 34:2248-2253, 2009

3. Andersen T, Christensen FB, Ernst C, Fruensgaard S, Østergaard J, Andersen JL, et al: The effect of electrical stimulation on lumbar spinal fusion in older patients: a randomized, controlled, multi-center trial. Part 1: functional outcome. Spine (Phila Pa 1976) 34:2241-2247, 2009

4. Andersen T, Christensen FB, Langdahl BL, Ernst C, Fruensgaard S, Ostergaard J, et al: Fusion mass bone quality after uninstrumented spinal fusion in older patients. Eur Spine J 19:2200-2208, 2010

5. Andersen T, Christensen FB, Niedermann B, Helmig P, Høy $\mathrm{K}$, Hansen ES, et al: Impact of instrumentation in lumbar spinal fusion in elderly patients: 71 patients followed for 2-7 years. Acta Orthop 80:445-450, 2009

6. Carreon LY, Glassman SD, Howard J: Fusion and nonsurgical treatment for symptomatic lumbar degenerative disease: a systematic review of Oswestry Disability Index and MOS Short Form-36 outcomes. Spine J 8:747-755, 2008

7. Dawson E, Bae HW, Burkus JK, Stambough JL, Glassman SD: Recombinant human bone morphogenetic protein-2 on an absorbable collagen sponge with an osteoconductive bulking agent in posterolateral arthrodesis with instrumentation. A prospective randomized trial. J Bone Joint Surg Am 91:16041613,2009

8. Dimar JR II, Glassman SD, Burkus JK, Pryor PW, Hardacker JW, Carreon LY: Clinical and radiographic analysis of an optimized rhBMP-2 formulation as an autograft replacement in posterolateral lumbar spine arthrodesis. J Bone Joint Surg Am 91:1377-1386, 2009

9. Dimar JR II, Glassman SD, Burkus JK, Pryor PW, Hardacker JW, Carreon LY: Two-year fusion and clinical outcomes in 224 patients treated with a single-level instrumented postero- 


\section{Part 17: Bone growth stimulators as an adjunct for lumbar fusion}

lateral fusion with iliac crest bone graft. Spine J 9:880-885, 2009

10. Dwyer AF: The use of electrical current stimulation in spinal fusion. Orthop Clin North Am 6:265-273, 1975

11. Fritzell P, Hägg O, Wessberg P, Nordwall A: Chronic low back pain and fusion: a comparison of three surgical techniques: a prospective multicenter randomized study from the Swedish Lumbar Spine Study Group. Spine (Phila Pa 1976) 27:11311141,2002

12. Ghogawala Z, Benzel EC, Amin-Hanjani S, Barker FG II, Harrington JF, Magge SN, et al: Prospective outcomes evaluation after decompression with or without instrumented fusion for lumbar stenosis and degenerative Grade I spondylolisthesis. J Neurosurg Spine 1:267-272, 2004

13. Kucharzyk DW: A controlled prospective outcome study of implantable electrical stimulation with spinal instrumentation in a high-risk spinal fusion population. Spine (Phila Pa 1976) 24:465-469, 1999

14. Lawlis GF, Cuencas R, Selby D, McCoy CE: The development of the Dallas Pain Questionnaire. An assessment of the impact of spinal pain on behavior. Spine (Phila Pa 1976) 14:511-516, 1989

15. Oishi M, Onesti ST: Electrical bone graft stimulation for spinal fusion: a review. Neurosurgery 47:1041-1056, 2000

16. Resnick DK, Choudhri TF, Dailey AT, Groff MW, Khoo L, Matz PG, et al: Guidelines for the performance of fusion procedures for degenerative disease of the lumbar spine. Part 1: introduction and methodology. J Neurosurg Spine 2:637-638, 2005

17. Resnick DK, Choudhri TF, Dailey AT, Groff MW, Khoo L, Matz PG, et al: Guidelines for the performance of fusion procedures for degenerative disease of the lumbar spine. Part 12: pedicle screw fixation as an adjunct to posterolateral fusion for low-back pain. J Neurosurg Spine 2:700-706, 2005

18. Resnick DK, Choudhri TF, Dailey AT, Groff MW, Khoo L, Matz PG, et al: Guidelines for the performance of fusion procedures for degenerative disease of the lumbar spine. Part 17: bone growth stimulators and lumbar fusion. J Neurosurg Spine 2:737-740, 2005

19. Rogozinski A, Rogozinski C: Efficacy of implanted bone growth stimulation in instrumented lumbosacral spinal fusion. Spine (Phila Pa 1976) 21:2479-2483, 1996

20. Rogozinski A, Rogozinski C, Cloud G: Accelerating autograft maturation in instrumented posterolateral lumbar spinal fusions without donor site morbidity. Orthopedics 32:809, 2009

21. Simmons JW Jr, Mooney V, Thacker I: Pseudarthrosis after lumbar spine fusion: nonoperative salvage with pulsed electromagnetic fields. Am J Orthop 33:27-30, 2004

22. Weinstein JN, Lurie JD, Tosteson TD, Zhao W, Blood EA, Tosteson AN, et al: Surgical compared with nonoperative treatment for lumbar degenerative spondylolisthesis. Four-year results in the Spine Patient Outcomes Research Trial (SPORT) randomized and observational cohorts. J Bone Joint Surg Am 91:1295-1304, 2009

Manuscript submitted March 27, 2014.

Accepted April 9, 2014.

Please include this information when citing this paper: DOI: 10.3171/2014.4.SPINE14326.

Address correspondence to: Michael G. Kaiser, M.D., Columbia University, Neurological Surgery, The Neurological Institute, 710 W. 168th St., New York, NY 10032.email: mgk7@columbia.edu. 